\title{
Digital Metering System of New Generation Smart Substation in China
}

\author{
Jingfen Bai ${ }^{1, a}$, Fantao Lin ${ }^{1}$, Xiangjiang Yang ${ }^{1}$, Jia Zuo ${ }^{1}$, Yingying Cheng ${ }^{2}$ and Chunping Yu ${ }^{1}$ \\ ${ }^{1}$ China Electrical Power Research Institute, 100192 Beijing ,China \\ ${ }^{2}$ Chongqing Electric Power Research Institute, 400000 Chongqing, China
}

\begin{abstract}
The new generation of smart substation is a summary and improvement of the earlier intelligent substations within the past 5 years. As a part of the substation operation function, the electric energy metering system must adapt to the requirements of the new generation smart substation which is more intelligent, full digitalization, networked and integrated. With the features of digitized, easy information sharing, and hardware shared, the digital measurement system is the inevitable choice for the new generation smart substation. This paper is the first literature to introduce the concept and structure of the digital metering system. The composition and technical characteristics of the new generation Smart Substation measurement system are introduced in detail.
\end{abstract}

\section{Introduction}

As one of the important parts in developing smart grid, the smart substation uses advanced, reliable, integrated, low- carbon and environmentally friendly equipments and design. With qualities of digitization of whole station information, networking of communication platform, standardization of information-sharing, integration of system function, compact design of structure, intelligent of highvoltage equipment and visualization of running status, smart substation can support power grid to realize real-time online analysis and control decision, and improve the operation reliability and economy.

The digitized, networked, standardized and integrated features of smart substation not only affect the design and configuration of secondary systems such as protection, measurement and control systems, but also affect the metering systems of the substation. Since 2009, the construction of smart substation has started in china [1], the early metering system of smart substation used traditional transformer, the digital level is not so high, and the integration with other secondary systems is not so deep, which limits the information-sharing between different services of the smart substation, and affects the level of intelligence. Compared with the earlier smart substations, the new generation smart substation puts forward higher requirements to intelligent, digitized and integrated level. The digital metering system should meet the overall requirements of the new generation smart substation with more comprehensive intelligent, digitization and integration.

\section{Digital Metering System}

\footnotetext{
${ }^{\mathrm{a}}$ Corresponding author : jfbai@epri.sgcc.com.cn
} 
With the feature of digitization, the digital metering system aims at metering and collecting electric energy in someone measuring point, it consists of digital electric energy metering device for power metering and acquisition terminal for power collection.

\subsection{Digital Electric Energy Metering Device}

The digital electric energy metering device consists of transformer, merging unit and digital input electricity meter, it is different from the traditional ones, and it adopts optical cable instead of electric cable to connect merging unit and digital input electricity meter, so the voltage drop caused by secondary cable can be reduced and result in composite error reduction [2-4]. This paper holds different views with previous records and believes that electronic transformer is not a distinguishing feature of the digital metering device, electronic transformer and traditional transformer are just in different way of sensing and different degree of digitization. In this paper, the digital electric energy metering device is classified into full digital and half digital in accordance with different signal sensors.

The signal sensing unit of full digital metering device is the electronic transformer. Between the transformer and the merging unit, and between the merging unit and the digital input electricity meter, voltage and current are transferred in the form of network packets which contain the discrete sampled values via different protocols, the structure is as shown in Figure 1: Synchronous Clock

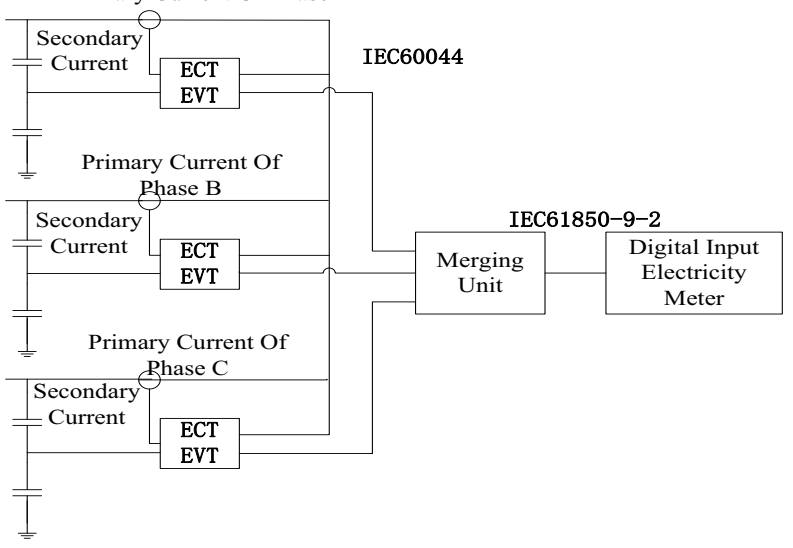

Figure 1. Structure of the full digital metering device.

The electronic transformer senses primary voltage and current, and transfers the sampled value to merge unit in the form of packet via the protocol of IEC60044, after the packet being collected and processed by merging unit, it will be assembled into new network packet via the protocol of IEC61850-9-2, and then be transferred to the digital input electricity meter of bay level by point-topoint or high -speed Ethernet mode, after a series of data processing and calculation, the electric energy accumulation of measurement point is completed.

The signal sensing unit of half digital metering device is the traditional electromagnetic transformer, traditional analog voltage and current signals are transferred between the transformers and merging unit, the structure is as shown in Figure 2:

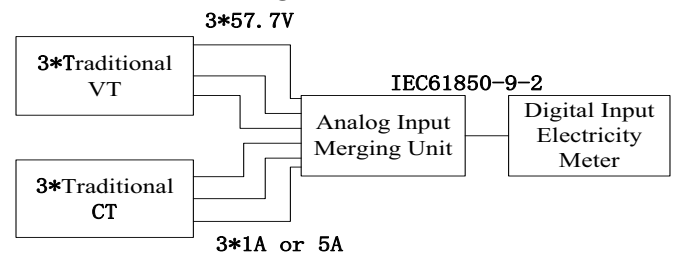

Figure 2. Structure of the half digital metering device. 
The traditional transformer converts high current and voltage into small signals of rated voltage $57.5 \mathrm{~V}$, and rated current $1 \mathrm{~A}$ or $5 \mathrm{~A}$, the analog input merging unit samples data uniformly. Then device transfers the signals and accumulates power in the same way as the full digital one.

\subsection{Electric Energy Acquisition Mode}

Smart substation is based on IEC61850 uniformly, in the early construction of smart substation, the digital input electricity meter only realized the digital input, but didn't completely follow the 61850 protocol to realize modelling and networking. The electric energy acquisition still used half duplex communication mode based on DL/T 645 communication protocol, which not only affects the internal sharing of data and the standardization of substation, but also affects the timeliness and integrity of data acquisition due to the limitation of the protocol itself, that the information related to primary power energy data and sampled value can't be transferred. The electric energy acquisition terminal of digital metering system acquires positive and negative active power, four quadrant reactive power, demand, real-time voltage/current/power, abnormal events and other information of the digital input electricity meter according to IEC61850-8-1MMS protocol, and then transfers the information to the main station in the form of packet according to IEC60870-5-102 protocol, which is as shown in Figure 3:

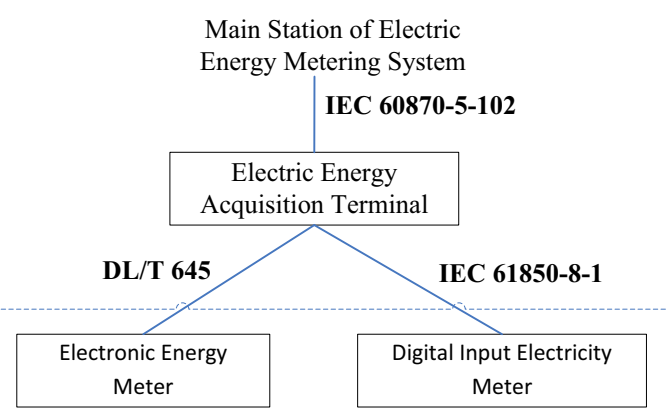

Figure 3. Acquisition structure of digital metering system.

The first version of IEC 61850 has only one logical node MMTR designed for electric energy, even the second version distinguishes three phase data from single phase data and just adds one logic node MMTN, which could not fully meet the requirement of electric energy data, so when use in China, it should be developed so as to adapt to the domestic business situation [5-9].

\subsection{Advantages of Digital Metering System}

(1)Digital signal is transferred by the digital metering system, so there is no error caused by secondary voltage drop due to the secondary cable connection, the error of the electric energy meter is reduced for the accumulation of electrical energy is a pure numerical calculation process, therefore, the composite error of the digital metering device only depends on the transformer and the merging unit, and thus will be greatly reduced.

(2)The digitization and networking features, the unified IEC 61850 protocol, the same data source and acquisition path with other secondary systems such as protecting, measuring and controlling system, all these make the digital metering system easy to realize data sharing and software intelligence, as well as hardware resource sharing.

(3)For full digital metering system, electronic transformer with the advantages of small size, unsaturated, wide band and so on, can expand the spectrum width of the system, and will be more suitable for harmonic measurement and impact load measurement. 


\section{Configuration Schemes of New Generation Smart Substation Electric Energy Metering System}

\subsection{Electric energy metering system of earlier smart substation}

The typical configuration of earlier smart substation electric energy metering system is shown as Figure 4:

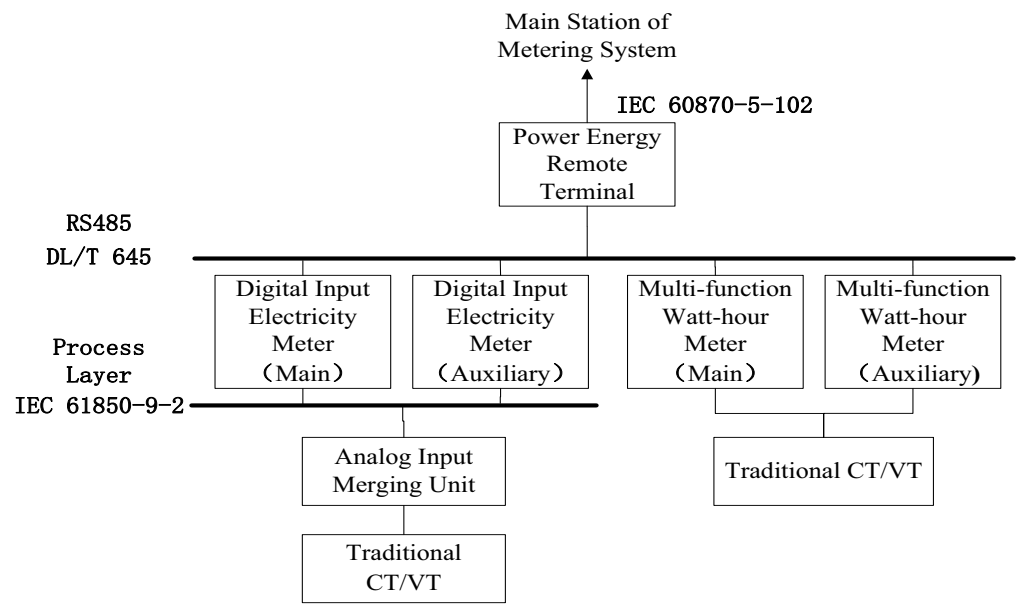

Figure 4. Typical configuration diagram of metering system.

Because of the electronic transformer exposed some stability and reliability problems in the early stages of development, the earlier smart substation did not use electronic transformer, but use the half digital metering device which was composed by analog input merging unit and digital electric energy meter. This composition can only be applied to the assessment measurement point where electric energy measurement is only used for the assessment of internal economic indicators or the assessment of power balance.

Measurement points for settlement belong to the category of national legal management. At present, in the case of merging unit and digital electric energy meter national metrological verification regulations have not been published, traditional electric energy measurement methods have still been used. The data acquisition method of assessment and settlement measurement points is to report power data according to DL/T 645 protocol by electric energy acquisition terminal through RS485 data bus.

\subsection{Electric Energy Metering System of New Generation Smart Substation}

Compared to the earlier smart substation, the new generation smart substation faces the demand of smart grid, places more emphasis on advanced features, integrated applications and interactive, strengthens the function applications of intelligent equipment, condition monitoring, advanced function of substation automation system and so on.

Compared with the earlier smart substation, the new generation smart substation has more prominent features of digitalization, networking. It uses electronic transformers as voltage and current sensing devices uniformly. Information accesses the substation automation system by merging unit through network, so as to realize the substation real-time information acquisition and security control functions. Compared to traditional transformer, electronic voltage/current transformer does not have many secondary windings for different function systems, the voltage/current digital sampler is included in the transformer, the merging unit only need to complete the merging and synchronization of the sampled value which from different voltage and current transformers. The output of the 
merging unit can also be the source of public information for the relay protection, measurement control and metrology devices, and so on.

In order to solve the problems such as overlapping network, multiple devices, taking multi screen cabinet, high construction cost, complex operation and maintenance in the earlier smart substation, one of the design targets of the new generation smart substation metering system is the integration with other secondary systems. And different services among metering, protection, and measurement control can share the network resources and the data source in order to simplify the network structure. Further, hardware resource can also be shared, the assessment measurement function which been undertook by independent meter originally can be incorporated into the measuring and controlling device, in such a way, the number of internal equipments and screen cabinets in station can be reduced, and the construction cost can be cut down. The configuration schemes of new generation smart substation measurement system are as follows.

Assessment measurement points share the output of merging units with other equipments in the station. Such kind of measurement points do not need independent digital electric energy meter, the metering function is integrated with other multifunction devices which have the same data source. For $110 \mathrm{kV}$ and above voltage levels, electronic transformers are used, measurement function is integrated in the digital input multi-function measuring and controlling device or device having integration of protection and measuring functions, for $10 \mathrm{kV} / 35 \mathrm{kV}$ voltage levels, analog transformers are used, measurement device is integrated in the multifunctional device. For metering integrated multifunction device, the positive/negative, active/reactive energy metering, demand metering, freeze, meter cleared, demand cleared, event logging and other basic measurement functions should be realized, the power pulse output port should be with, which facilitates the detection of energy measurement error, algorithm of energy measurement was improved to make sure the measurement and control device could meet the requirements of $0.5 \mathrm{~S}$ level, and meets the electric energy metering accuracy need of assessment measurement.

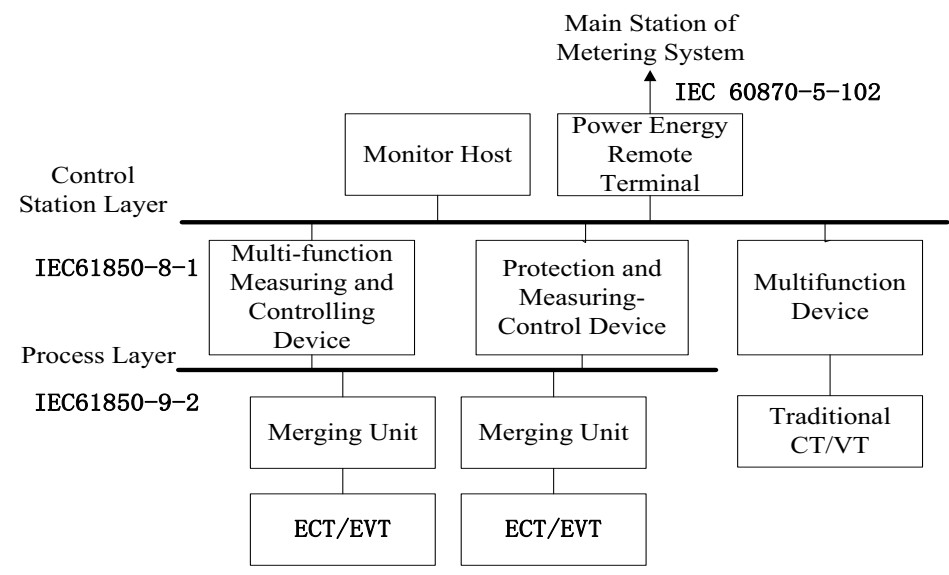

Figure 5. Assessment measurement point configuration scheme.

For settlement measurement points, the relevant equipment verification regulations haven't yet been published at present, the traditional transformer and electricity meter are configured independently according to DL/T 448. If the tracing and transmitting system of the digital electric energy meter, electronic transformer, merging unit and other equipment is established in the future, the digital metering system will be used for trade settlement, according to the reliability of different components and the metering needs of settlement measurement points, the following settlement measurement point configuration scheme is predicted, as shown in Figure 6: 


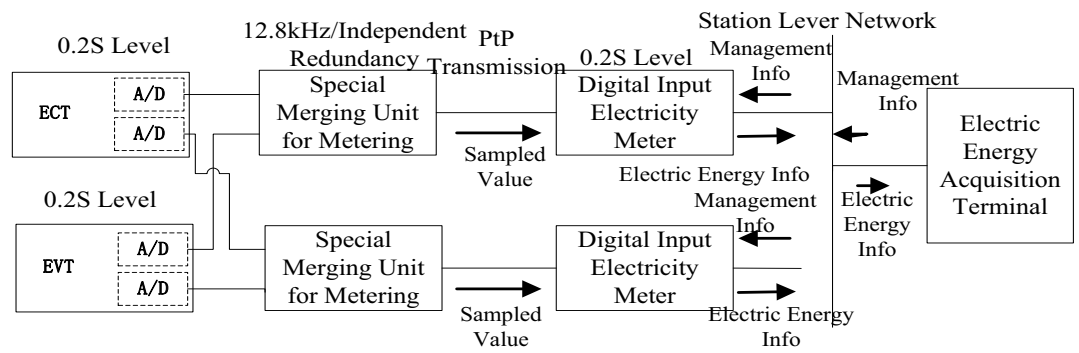

Figure 6. Advise of settlement measurement points configuration scheme.

The A / D sampling modules of ECT/EVT, merging unit, and digital input electricity meter are all electron devices, the reliability of which is lower than the transformer sensor modules. Therefore, the main/standby redundant configuration method needs to be prepared. The bay level needs to be equipped with special merging unit for metering with hardware protection function. The sampling data of merging unit is transferred point-to-point to the digital input electricity meter, which guarantees the security and independence of settlement measurement system. The electronic current transformer, the accuracy level of which is $0.2 \mathrm{~S}$, can meet the needs of small current measurement. Meanwhile, the merging unit, the output sampling frequency of which is $12.8 \mathrm{kHz}$, can support less than 40th harmonic metering. In addition to meeting the basic energy metering, the digital input electricity meter should also have the harmonic metering function, moreover, coordinating with other software functions such as measurement data timing freeze so as to ensure that the settlement measurement system is absolutely safe, reliable and fair.

There is a kind of measurement point that usually for assessment, but in special circumstances may be transformed into a settlement point. For example, if in the opposite side is the settlement point, in this side is such kind of measurement point, it will be transformed into settlement one when the device in the opposite side is invalid. For this kind of measurement point, the transformer, merging unit, etc, are designed according to the assessment measurement point, but the digital input electricity meter should be configured independently, as shown in Figure 7:

With the exception of the multi-function Walt-hour meter configured on settlement point still using DL/T 645, the digital input electricity meter and all kinds of measurement integration devices all use extended IEC 61850-8-1 protocol to acquire electric energy.

The devices such as multi-function measuring and controlling device, multifunction device, digital input electricity meter, merging unit, and electronic transformer, should meet the requirements of corresponding accuracy level in accordance with DL/T 448.

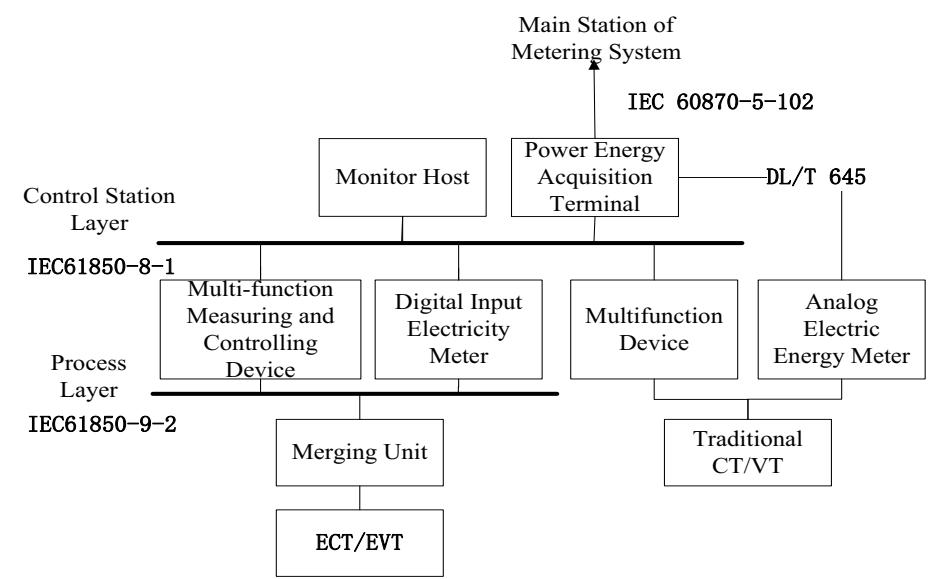

Figure 7. Assessment measurement point transformed into settlement point configuration scheme. 


\subsection{Metering Communication Model of New Generation Substation based on IEC 61850}

Up till now, there is no metering communication data model based on IEC 61850 both in China and abroad. In order to achieve the standardization of measurement data, and to guarantee the interoperability and exchange of each equipment in the system, the following extension is carried out based on the analysis of the functions such as electric energy metering, demand metering, freeze, event reporting, and time consumption metering, as shown in Figure 8:

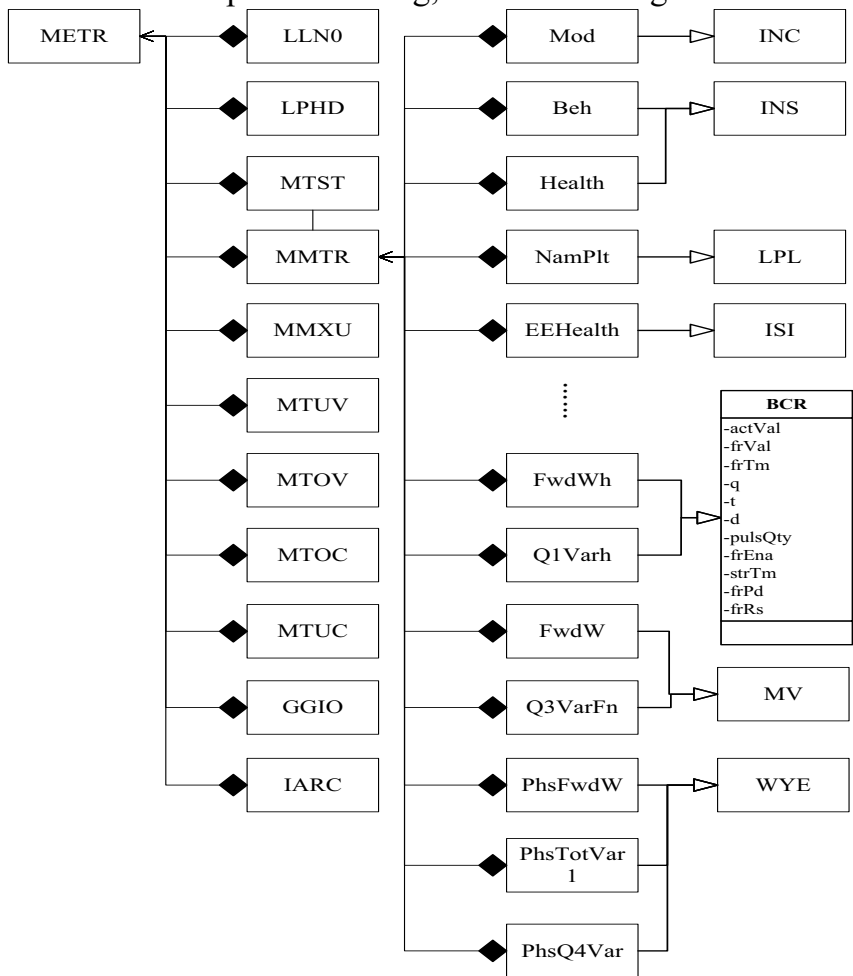

Figure 8. Energy Metering Communication Model based on IEC 61850

(1)The IEC61850-7-4 logic node MMTR can be extended for positive and negative active power metering, four quadrant reactive power metering and demand metering (MMTR for three-phase electric energy meter, and MMTN for single-phase electric energy meter). Different cases should be created for electric energy metering and demand metering, for instance, case MMTR1 for electric energy metering and case MMTR2 for demand metering, different cases are distinguished by Arabia digital suffix.

(2)The original logic node MMXU is used to measure voltage, current and so on (MMXU for three-phase electric energy meter, and MMXN for single-phase electric energy meter).

(3)The loss of voltage, phase failure and loss of current alarm limits for metering are different from the protection and control alarm limits, the MTUV、MTOV、MTUC、MTOC are created so as to monitor alarm. Different cases are created for different event alarms, even if they belong to the same type, for instance, case MTUV1 for under voltage, and case MTUV2 for loss of voltage, different cases are distinguished by Arabia digital suffix.

(4)The original logic node GGIO is used to report alarm events which needn't to be set or customized, such as abnormal sampling, electric energy meter failure, power fail, and voltage negative phase sequence, etc.

(5)The original logic node IARC is used to record programming events such as time zone /time consumption table programming, demand interval programming, meter cleared, demand cleared, event cleared, cover opening, and end button box opening, etc. 
(6)The logic node MTST is created for saving and recording time zone table.

(7)The meter asset information, such as rated voltage, rated current, active/reactive power combination status word, active power pulse constant, and reactive power pulse constant, is taken as the data object of extended logic node LLN0.

\subsection{Characteristics of the New Generation Smart Substation's Metering System}

(1)Based on the design concept of unified data source and simplifying communication network in station, the full digital measurement system and the technical solution of integrating measurement service with other systems have been adopted, by incorporating the special communication network into the public communication network which based on IEC61850, data source and hardware resource sharing among measurement service and other systems will be realized to simplify the secondary system, that lays a good foundation for intelligent enhancing.

(2)According to the demand of measurement service, the measuring points can be sorted into three types: the assessment measurement points, the settlement measurement points and the assessment measurement points which can be transformed into settlement points in special circumstances. Different types of measurement points need different implementation schemes and different technical conditions.

(3)The IEC61850 node model and service model for the measurement and management of new generation smart substation have been established, furthermore, real time record and easy call after event of large number of frozen data have been achieved by use of IEC61850 file services, meanwhile, real-time active reporting of abnormal events has also been achieved by use of IEC61850 reporting services.

\section{Measurement Technology of the New Generation Smart Substation Metering Device}

Integrated equipments with measuring functions, such as digital input electricity meter, merging unit, multi-function measuring and controlling device are all new type of measurement devices, the using environment and application features are very different from the original analog electric energy meter[10-11]. On the basis of focusing on the test of basic error and measurement function, in the professional testing of measurement equipments in the new generation smart substation demonstration project, China Electric Power Research Institute adds anomaly detection in the network pressure and data packet loss situation, the added test items are as follow:

(1)The sampling value input interface receiving power test is to detect the receiving power range of the sampling value input port of the testing device. The optical attenuation meter is connected in series between a digital electric energy meter calibrator and the digital input electricity meter under test, slowly increase the attenuation of the optical attenuation meter from the beginning of 0 until the alarm lamp of the digital input electricity meter is lit, then pull off the tail fiber of digital input electricity meter, and access it to the optical power meter, read the power value at this time as the result of measurement, the test block diagram is shown as Figure 9:

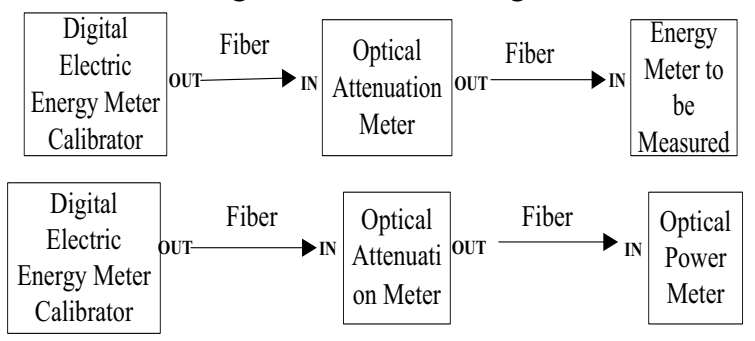

Figure 9. Sampling value input interface receiver power test block diagram. 
(2)The sampling data loss effect test is to validate whether the error of energy meter meets the requirement of the grade index in case of abnormal sampling data. The digital input electricity meter testing device, in accordance with the lost point rate that not exceed the grade index, outputs exception signals of sampled value in a loop continuously, and records the measurement error, the error should be in accordance with the grade index.

(3)The network storm effect test is to validate whether the measurement performance of energy meter meets the requirement of the grade index when the sampled value input interface is in large network pressure. The digital input electricity meter testing device sends sampled value signals via switch port 1, the network tester join the broadcast storm to switch port 3 via port 2, the measurement error of the digital input electricity meter being tested should be in accordance with the grade index, the test block diagram is shown as Figure 10:

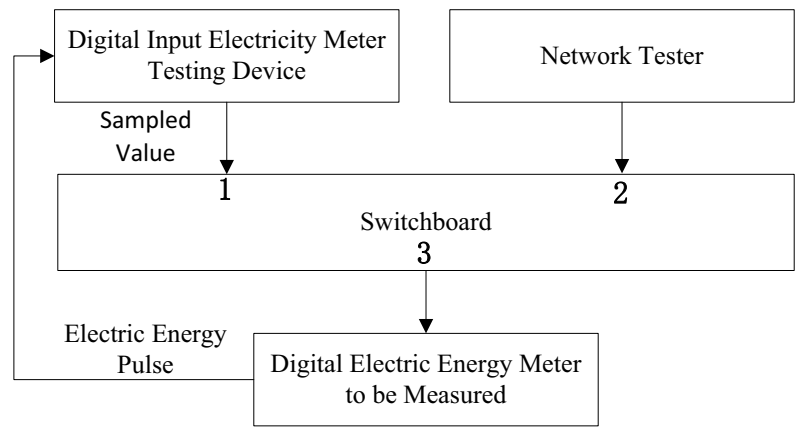

Figure 10. Network storm effect test block diagram

(4)The protocol conformance check includes sampled value protocol conformance test, configuration file check, and IEC 61850 communication function dynamic check, etc. The block diagram of dynamic check is shown as Figure 11. The testing software controls the digital electric energy meter calibrator with the pre edited test case by simulating normal state and different abnormal events according to the abnormal device duration, the output voltage and current value, the meter calibrator receives the MMS packet returned by the tested power meter, and analyses the MMS packet response node, so as to judge whether the tested power meter could respond, whether the action is consistent with the preset result of the test case, and whether the return time meets the standard requirements .

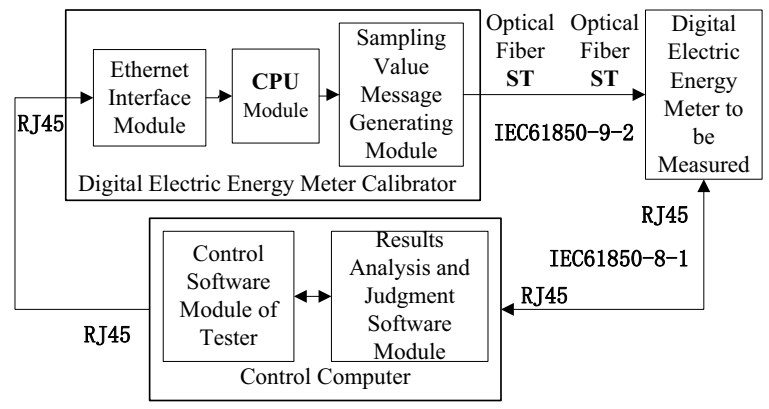

Figure 11. Block diagram of the IEC 61850 protocol conformance test system.

\section{Conclusion}

The new generation smart substation is more digitized, networked, integrated and intelligent. To adapting these improvements of the new smart substation, an even bigger evolution is carried on the internal metering system of the substation. On the basis of introducing the digital metering system, this article provides details of the system structure and related equipment detection technology of the 
digital metering system of the new smart substation. The new digital metering system is a business integration and promotion of the ordinary digital metering systems. The new metering devices, such as the testing and control device with metering function, and the new metering technologies, such as IEC 61850 meter reading application, are used for the first time. Whether these new devices and new technologies can meet the reliability, stability and security requirements of the system needs to be summarized and found in the operation process of the substation. At present, the digital metering system cannot be used as the basis for trade settlement yet. However, with the establishment of national digital metering system, in the near future, the digital metering system will be applied to all voltage levels and all types of measurement points thanks to its unique technical characteristics.

\section{Acknowledgement}

Supported by science and technology project of China State Grid Corp.( research of the on-site calibration technology for digital metering device and monitoring system of the new generation smart substation)

\section{References}

1. Q.Y. Zhang, Guizhou. Electr. Pow. Technol 14, 1(2011)

2. S.J. Wu, J.F. Bai, H.B. Yu, et al, Power. Syst. Technol 38, (supplement 1) (2014)

3. L.M. Cai, G.H. Liu, Electr. Pow 44, 4 (2011)

4. K. Jiang, T. Shang, B. Chen, et al, J. Zhongyuan. Univers. Technol 23, 2(2012)

5. J.F. Bai, F.T. Lin, S.J. Wu, Y.H. Xu, et al, ICMPMT 2013

6. L. He, IEC 61850 Application (CEPP, 2012)

7. IEC 61850 Communication networks and systems in substations (2003)

8. Y.L. Li, G. Li, Power. Syst. Technol 34, 4 (2010)

9. J.M. Zhang, S.T. Jiang, N.Z. Jin, et al, Automat. Electr. Pow. Syst 34, 11(2010)

10. Y. Xiao, B. Jiang, W. Zhao, B. Ai, S.L. Huang, Y. Tang, X.S. Chen, Electr. Meas. Instr 51,1(2014)

11. Q. Li, S.H. Zhang, Y.B. Lu, D. Han, H.L. Hu, H. Li, D.Y. Li, Electr. Meas. Instr 47, 10(2010) 